\title{
An investigation of the facilitation of simple auditory reaction time by predictable background stimuli
}

\author{
DAVID S. EMMERICH, DEBORAH A. FANTINI, and WOLFGANG ELLERMEIER \\ State University of New York, Stony Brook, New York
}

Two experiments explored a surprising result reported by Emmerich, Pitchford, and Becker (1976): Simple reaction time (RT) to an auditory stimulus can be facilitated by the presence of a tonal background (or masker). In the first experiment, simple RT to a tonal signal was investigated for a variety of background frequencies and loudness levels, and significant facilitation of RT was found for low levels of the background. In the second experiment, no evidence of facilitation was found when the background stimulus was a randomly varying narrow-band noise, although evidence for facilitation was again found with a constant tonal background.

To learn more about the sensory processing performed by the auditory system, researchers have investigated the responses of listeners to combinations of sinusoidal stimuli in a wide variety of studies. For example, since the time of Wegel and Lane (1924), the study of the influence of one tone on the threshold of another, or tonal masking, has been an active area of investigation in auditory psychophysics. Fletcher and Munson (1933) and numerous subsequent researchers have extended this research to the study of suprathreshold stimuli in investigations of the perceived loudness of tonal complexes. Other suprathreshold phenomena such as beats and combination tones have also received much attention. Surprisingly, however, few researchers interested in the sensory processing of suprathreshold combinations of tonal stimuli have investigated simple reaction time (RT), despite the fact that it is a sensitive measure and would be expected-since the RT task makes relatively few cognitive demands on the subject - to be closely related to basic underlying sensory processes.

Chocholle and his co-workers (Chocholle, 1972; Chocholle \& Greenbaum, 1965, 1966; Chocholle \& Saulnier, 1969) carried out a series of simple auditory RT studies in which the signal was a $1000-\mathrm{Hz}$ tone presented in a background of either one or two other tones. As one would expect from the literature on masking and loudness, these studies found that the presence of more intense background stimuli led to an increase in RT to the $1000-\mathrm{Hz}$ signal tone. When Emmerich, Pitchford, and

Portions of this research were presented at the 108th meeting of the Acoustical Society of America, Minneapolis, Minnesota, October 1984. The present address of Deborah A. Fantini is MRC Institute of Hearing Research, University of Nottingham, University Park, Nottingham NG7 2RD, England. The present address of Wolfgang Ellermeier is Physiologisches Institut der Universität Würzburg, Röntgenring 9, D-8700 Würzburg, West Germany. Requests for reprints should be sent to David S. Emmerich, Department of Psychology, State University of New York, Stony Brook, NY 11794.
Becker (1976) extended this research to a situation in which there was a wide separation between the frequency of a signal tone and that of a tonal masker, however, a counterintuitive result was obtained. In this case, rather than slowing down responses to the signal tone, the tonal background actually led to a significant facilitation of RT.

The present research was undertaken with two goals in mind. The first, addressed in Experiment 1, was simply to investigate RT to tones presented in tonal backgrounds for a wide range of different background tones, in order to explore the generality of the surprising facilitation of RT observed by Emmerich et al. (1976). The second goal, addressed in Experiment 2, was to examine a possible mechanism that might underlie this facilitation.

\section{EXPERIMENT 1}

In this experiment, subjects were asked to respond to brief $2000-\mathrm{Hz}$ signal tones presented in tonal backgrounds that varied in both frequency and intensity over a relatively wide range. The frequencies of the tones were spaced approximately logarithmically and were chosen from a range in which the loudness of equal-intensity tones changes very little with frequency.

\section{Method}

Subjects. Four volunteers with clinically normal hearing in the frequency range used in the experiment served as subjects.

Apparatus. General Radio 1310A oscillators were used to produce tonal stimuli, and a Grason-Stadler $455 \mathrm{C}$ white-noise generator was used to produce noise. Stimuli were presented by means of an electronic switch and Telephonics TDH-39 earphones. The subjects were seated in a sound-attenuating chamber.

Procedure. A 200-msec burst of white noise was presented to the subject's right ear at a spectral level of 10-dB SPL to signal the beginning of each trial. Immediately following this warning signal, the background stimulus was presented to the left ear and remained for the rest of the trial. After a randomly chosen foreperiod of $2.00,2.25$, or $2.50 \mathrm{sec}$, the signal tone was added to the background in the left ear. The signal tone always had a frequency of 
$2000 \mathrm{~Hz}$, a sound pressure level of $50 \mathrm{~dB}$, and a duration of $50 \mathrm{msec}$; it was switched with a rise/decay time of $2.5 \mathrm{msec}$. The subjects were instructed to keep a finger poised on a telegraph key and to press this key as quickly as possible after presentation of the signal tone. RT was measured in milliseconds from the onset of the signal tone. ${ }^{1}$ Following the subject's response, the background stimulus was turned off, and a 1.5-sec intertrial interval was started.

The background stimulus on a given trial was chosen randomly from four equally likely alternatives: silence, or a tone at the 30 , 50 , or $70 \mathrm{~dB}$ loudness level. The background frequencies employed were $1000,1250,1600,2500$, and $3150 \mathrm{~Hz}$ and were counterbalanced across blocks of trials. Since loudness changes relatively little with frequency over this range, the various backgrounds were very nearly equated in sound pressure level as well as in loudness level: the largest change in sound pressure level needed to equate loudness levels was $3 \mathrm{~dB}$ (Fletcher \& Munson, 1933).

Following an initial practice session, each subject participated in eight 1-h experimental sessions. The subjects were run alternately in pairs, with one member of the pair resting while the other completed a block of 55 trials. In each session, each subject completed a single block of trials for each of the five background frequencies, with the frequencies presented in a random order. Thus, each subject received 8 different orders of the background frequencies. In all, 32 randomly chosen orders were used. The first 5 trials of each block were considered practice and were not included in the data analysis. Trials with RTs shorter than $100 \mathrm{msec}$ or greater than $500 \mathrm{msec}$ were also excluded from the analysis. Though such trials were infrequent ( 141 trials out of a total of 8,000 ), their exclusion undoubtedly makes the present data unsuitable for the development of precise quantitative models of the underlying processes.

\section{Results and Discussion}

A preliminary analysis was conducted to determine if there were practice effects over the eight sessions of the experiment, and, if so, whether they interacted with the frequency of the tonal background. This analysis indicates that there were significant differences in mean RT across sessions $[F(7,21)=427.00, p<.001]$. There was no interaction with background frequency $[F(28,84)<1]$, however.

The mean RT, averaged across experimental conditions, was $176.0 \mathrm{msec}$ in the first session of the experiment. Following this session, there was an overall decline in mean RT across the eight sessions of the experiment, with a mean RT of $170.1 \mathrm{msec}$ obtained in the final session. This overall decline was somewhat obscured by a tendency for mean RT to increase and decrease in alternate sessions of the experiment after the first session. The linear trend component, reflecting the overall decrease in $\mathrm{RT}$ across the experiment, is statistically significant $[F(1,21)=$ $16.71, p<.001]$. Two higher order trend components, the sixth- $[F(1,21)=5.68, p<.05]$ and seventh-order $[F(1,21)=16.94, p<.001]$ components, are also statistically significant, presumably as a result of the way in which RT tended to alternately increase and decrease across sessions. Since there was no interaction of session with background frequency, the data were collapsed across experimental session in subsequent analyses.

A second preliminary analysis investigated the effects of foreperiod duration. The mean RT for stimuli presented after the 2-sec foreperiod was $181.3 \mathrm{msec}$, that for stimuli presented after the 2.25 -sec foreperiod was $170.1 \mathrm{msec}$, and that for stimuli presented after the $2.5-\mathrm{sec}$ foreperiod was $166.3 \mathrm{msec}$. An analysis of variance indicates that these means differ significantly $[F(2,6)=14.48$, $p<.005]$, presumably indicating that responses could be made more quickly with decreasing uncertainty as to when the signal would be presented. There was also a tendency for more responses to be excluded from the analysis as the foreperiod increased: 37,38 , and 66 trials were excluded on trials with short, medium, and long foreperiods, respectively. These numbers of excluded trials do not differ significantly, however (Friedman test, $p>.30$ ).

The foreperiod-duration variable did not interact significantly with either background frequency $[F(8,24)<1]$ or background level $[F(6,18)=1.07, p=$ $.41]$, and there was no significant three-way interaction $[F(24,72)=1.57, p=.07]$ among these variables. Accordingly, the data were collapsed across foreperiod duration in subsequent analyses.

The mean RT values for the $2000-\mathrm{Hz}$ signal tones presented in the backgrounds of each frequency and loudness level were calculated for each of the 4 subjects. The means and standard deviations of these four values for each combination of background frequency and loudness level are presented in Table 1. The means in Table 1 are each based on approximately $\mathbf{4 0 0}$ trials. An analysis of variance indicates that there is a significant main effect of both background level $[F(3,9)=4.74, p=.029]$ and background frequency $[F(4,12)=4.00, p=.027]$ on RT. In addition, there is a significant interaction between background level and frequency $[F(12,36)=5.45, p<.001]$, indicating that the change in RT with level differs as a function of frequency.

Table 1 shows that for all background frequencies, RT is faster when signal tones are added to the 30- $\mathrm{dB}$ background than when they are presented in quiet, thus replicating the surprising facilitation of RT reported by Emmerich et al. (1976). The difference between RT in the quiet background and RT in the lowest background level is statistically significant $[F(1,3)=309.40, p<$ $.001]$. As the loudness level of the background is increased, however, mean RT tends to become slower and does not differ significantly from RT in the quiet for either the 50 -dB background $[F(1,3)=6.82, p>.05]$ or the 70 -dB background $[F(1,3)=.963, p>.05]$.

The significant interaction of loudness level and frequency is presumably due to the fact that the decrease in facilitation of RT as loudness level is increased above $30 \mathrm{~dB}$ is much more prominent for background frequencies below the signal frequency than for those above it. In fact, for these lower frequencies, RT is slower for the 70-dB loudness level than it is in the quiet: if anything, facilitation has turned into inhibition or masking. The data of Table 1 are reminiscent of the literature on auditory masking in which it has been well established since the time of the classic study of Wegel and Lane (1924) that low frequencies mask high frequencies much more than the converse, that masking increases with intensity of the 
Table 1

Mean Reaction Times and Standard Deviations (in Msec) to 2000-Hz Signals Presented in Backgrounds Differing in Frequency and Loudness Level

\begin{tabular}{|c|c|c|c|c|c|c|c|c|}
\hline \multirow{3}{*}{$\begin{array}{l}\text { Background } \\
\text { Frequency }(\mathrm{Hz})\end{array}$} & \multicolumn{8}{|c|}{ Background Level } \\
\hline & \multicolumn{2}{|c|}{ Quiet } & \multicolumn{2}{|c|}{$30 \mathrm{~dB}$} & \multicolumn{2}{|c|}{$50 \mathrm{~dB}$} & \multicolumn{2}{|c|}{$70 \mathrm{~dB}$} \\
\hline & $M$ & $S D$ & $M$ & $S D$ & $M$ & $S D$ & $M$ & $S D$ \\
\hline 1000 & 172.8 & 17.8 & 169.9 & 17.4 & 169.9 & 15.7 & 173.0 & 16.0 \\
\hline 1250 & 174.4 & 16.5 & 166.6 & 15.3 & 168.2 & 14.5 & 179.3 & 19.5 \\
\hline 1600 & 174.9 & 12.5 & 168.4 & 12.3 & 173.9 & 10.8 & 191.2 & 20.5 \\
\hline 2500 & 173.3 & 16.7 & 171.2 & 15.5 & 171.9 & 14.2 & 172.7 & 15.5 \\
\hline 3150 & 179.1 & 18.1 & 172.5 & 18.1 & 169.9 & 13.8 & 171.7 & 15.8 \\
\hline
\end{tabular}

masker, and that masking increases as the frequency separation between masker and signal is decreased. As is also consistent with the literature, when the $1600-\mathrm{Hz}$ background was presented at a loudness level of $70 \mathrm{~dB}$, subjects reporting hearing a lower frequency combination tone rather than the $2000-\mathrm{Hz}$ signal tone heard on other trials. The apparent change in frequency may have contributed to the slowness of RT in this condition.

A possible explanation for the pattern of results observed in this experiment is suggested by the physiological literature. Physiological studies of the phenomenon of two-tone suppression (e.g., Abbas \& Sachs, 1976; Sachs \& Kiang, 1968) show that the ongoing firing rate of an auditory nerve fiber that is responding to the presence of a tone can be decreased by the addition of a second tone to the first. Such a decrease in the ongoing firing rate in neurons at some level in the auditory system may provide a useful cue to the fact that the second tone has been added. If so, a subject in an experiment such as ours would have two potential cues to the presentation of the signal tone when a background tone is already present: a decrement in the ongoing firing rate of fibers, which were already responding to the presence of the background tone, and the excitation of new fibers, which would begin firing as a result of the addition of the signal tone. When no background tone is present, however, only the excitation of new fibers would be available as a cue to indicate the presentation of the signal tone. To the extent that the two cues available when a background tone is present are more useful than the single cue available in its absence, subjects should be able to detect the presentation of the signal tone more quickly in this background than in the quiet one. Only when the background tone is sufficiently intense to greatly diminish the effects of the presentation of the signal tone would its presence be a liability.

If changes in the ongoing activity of fibers responding to the steady tonal background are responsible for the facilitation of RT reported by Emmerich et al. (1976), and shown in Table 1, then less facilitation should be expected in the presence of a background that exhibits continual random fluctuations in level. That is, a steady tonal background of long duration should lead to the establishment of a relatively constant rate of firing in fibers respon- sive to this background, which in turn should make changes in firing rate relatively easy to detect. In contrast, in a background that varies randomly in level, any modulation of the ongoing firing rate that occurs as a result of the addition of the signal should be harder to detect due to the random variation in firing that is already present. The second experiment investigated this possibility.

\section{EXPERIMENT 2}

In this experiment, subjects were asked to detect tonal signals presented either in a quiet background, in a constant tonal background, or in a randomly varying background. On the basis of Emmerich et al.'s (1976) results, as well as those of Experiment 1, it was expected that RT would be faster in the constant tonal background than in the quiet background. Of greater interest was the randomly varying condition: if the analysis presented above was correct, then less facilitation should be observed in this condition.

\section{Method}

Subjects. Twenty volunteers with clinically normal hearing in the frequency range used in the experiment served as subjects.

Apparatus. In addition to the apparatus used in Experiment 1, a second noise generator and a narrow-band filter were used to create a randomly varying background. When white noise is passed through a narrow-band filter, the resulting output approximates a sinusoid which varies randomly in frequency and intensity. The filter was centered at a frequency of $989 \mathrm{~Hz}$ and was $3 \mathrm{~Hz}$ wide at the halfpower points.

Procedure. The trial sequence used was the same as that employed in Experiment 1. Again, the background condition was instituted during a variable foreperiod of $2.00,2.25$, or $2.50 \mathrm{sec}$. However, only three backgrounds (chosen at random for each trial) were used in the second experiment: a quiet background, a $989-\mathrm{Hz}$ tonal background, and a narrow-band noise background. The background tone had a sound pressure level of $65 \mathrm{~dB}$. The average overall sound pressure level of the narrow-band noise was also $65 \mathrm{~dB}$.

Signal tones were presented monaurally at $45 \mathrm{~dB}$ SPL, had a duration of $50 \mathrm{msec}$, and were switched with a rise/decay time of $2.5 \mathrm{msec}$. For half of the subjects, the signal frequency was $500 \mathrm{~Hz}$, and for half it was $2000 \mathrm{~Hz}$.

Subjects were again run in pairs, with one member of the pair resting while data were being obtained from the other. The subjects changed places after each block of 55 trials until each subject 
had completed one practice block and six data blocks. The first 5 trials of a block were considered practice and were not analyzed. Trials on which RTs were shorter than $100 \mathrm{msec}$ or longer than $500 \mathrm{msec}$ were replaced. Such occurrences were infrequent: 82 of the 3,000 trials obtained with $500-\mathrm{Hz}$ signals and 91 of the 3,000 trials obtained with $2000-\mathrm{Hz}$ signals were replaced in this manner.

\section{Results and Discussion}

Mean RT values were computed for each combination of foreperiod duration and background condition for both the subjects who had received the $500-\mathrm{Hz}$ signal tones and those who had received the 2000-Hz signal tones. An analysis of variance indicated that for the $500-\mathrm{Hz}$ signals, RT differed significantly as a function of foreperiod duration $[F(2,18)=4.81, p<.02]$. As in Experiment 1 , mean RT decreased as foreperiod increased: the means for the 2.00-, 2.25-, and 2.5-sec foreperiods were 258.6, 251.2, and $248.4 \mathrm{msec}$, respectively. Again, there was no significant interaction of foreperiod duration with the type of background $[F(4,36)<1]$, and the numbers of trials replaced in the different foreperiod conditions $(29,23$, and 30 trials) did not differ significantly (Friedman test, $p>.5$ ).

Similarly, for the 2000-Hz signals, RT also differed significantly as a function of foreperiod duration $[F(2,18)$ $=8.71, p<.002$ ], decreasing from $252.6 \mathrm{msec}$ for the 2 -sec foreperiod to $245.2 \mathrm{msec}$ for the 2.25 -sec foreperiod and to $242.9 \mathrm{msec}$ for the $2.5-\mathrm{sec}$ foreperiod. There was no significant interaction of foreperiod duration with the type of background $[F(4,36)=.182, p=.146]$, and the numbers of trials replaced in the short, medium, and long foreperiod conditions $(30,33$, and 28 trials) did not differ significantly (Friedman test, $p>.5$ ).

In addition to varying with foreperiod, mean RT also varied significantly as a function of background condition, both for subjects who had received the $500-\mathrm{Hz}$ signals $[F(2,18)=29.42, p<.001]$, and for those who had received the $2000-\mathrm{Hz}$ signals $[F(2,18)=9.37, p<.001]$. The means, collapsed across foreperiod, were computed for each background condition for each of the 10 subjects who had received each signal frequency. The means (and standard deviations) of the 10 individual scores in each condition are presented in Table 2 . These means are each based on approximately 1,000 trials.

Planned comparisons were carried out to determine in which conditions RT differed significantly from RT in the quiet. These comparisons indicate that for $500-\mathrm{Hz}$ signals, RT is significantly faster with a constant tonal back-

Table 2

Mean Reaction Times and Standard Deviations (in Msec) to Signal Tones Presented in Different Backgrounds

\begin{tabular}{|c|c|c|c|c|c|c|}
\hline \multirow{3}{*}{$\begin{array}{l}\text { Frequency of } \\
\text { Signal Tone (Hz) }\end{array}$} & \multicolumn{6}{|c|}{ Background } \\
\hline & \multicolumn{2}{|c|}{ Quiet } & \multicolumn{2}{|c|}{ Tonal } & \multicolumn{2}{|c|}{$\begin{array}{l}\text { Narrow-band } \\
\text { Noise } \\
\end{array}$} \\
\hline & $M$ & $S D$ & $\bar{M}$ & $S D$ & $M$ & $\overline{S D}$ \\
\hline 500 & 244.9 & 27.7 & 234.6 & 29.5 & 277.7 & $\overline{35.5}$ \\
\hline 2000 & 248.3 & 43.3 & 235.0 & 35.3 & 256.7 & 36.9 \\
\hline
\end{tabular}

ground than with the quiet background $[F(1,18)=3.16$, $p<.05$, one-tailed test]. In addition, for these signals, not only is there no facilitation of RT by the background of narrow-band noise, but RT is significantly slower in this background than it is in the quiet $[F(1,18)=31.06$, $p<.001$ ]. For the $2000-\mathrm{Hz}$ signals, the facilitation by the constant tonal background is also statistically significant $[F(1,18)=6.74, p<.01$, one-tailed test $]$, but the apparent slowing of RT by the narrow-band noise is not $[F(1,18)=2.89, p>.05]$.

Thus, in the second experiment, there is again evidence of facilitation of RT by the presence of the constant tonal background, but no evidence at all for such facilitation by the randomly varying background of narrow-band noise, either for signals lower or for those higher in frequency than the noise band. These results are consistent with the notion that the facilitation of RT observed in Experiment 1, and in Emmerich et al. (1976), is due to the modulation of ongoing neural activity (initiated by the tonal background) which occurs as a result of signal presentation.

The above discussion brings to mind analogous discussions in the masking literature of the cues used by subjects given the task of detecting faint sinusoidal signals presented in various backgrounds. For example, Wegel and Lane (1924) indicated that, for small separations between the frequency of the signal (or its overtones) and the frequency of a sinusoidal masker, subjects had reported that the presence of the signal was detected by hearing beats with the masker rather than by hearing the signal itself. Similarly, Green (1967) suggested that a random noise background was a more effective masker of a tonal signal than was a sinusoidal masker at the signal frequency, since in the latter case the subject could make use of the cue of a change in the masker envelope to detect the addition of the signal. This cue would not be available, of course, with the random noise background.

\section{SUMMARY AND CONCLUSIONS}

The data presented above are consistent with the following generalizations. The presence of a tonal background of low intensity can facilitate RT to a tonal signal of moderate intensity. It seems likely that this facilitation of RT is accomplished by subjects' monitoring changes in the ongoing neural activity initiated by the tonal background while simultaneously listening for the addition of the signal tone. As the intensity of the tonal background increases, this facilitation decreases and, for background frequencies lower than the signal frequency, can turn into slowing. The rate at which RT changes with intensity appears to depend on the relative frequencies of the background and the signal.

\section{REFERENCES}

AbBas, P. J., \& SACHS, M. B. (1976). Two-tone suppression in auditory nerve fibers: Extension of a stimulus-response relationship. Journal of the Acoustical Society of America, 59, 112-122. 
Chосноlle, R. (1972). Effets de masque homolatéraux et contralatéraux, totaux et partiels, sur un son pur de deux autres sons purs placés de part et d'autre du premier. Acustica, 27, 267-277.

Chocholle, R., \& Greenbaum, H. (1965). Les temps de réaction aux sons partiellement masqués. Journal de Physiologie, Paris, 57, 588. (Abstract)

Chocholle, R. \& Greenbaum, H. (1966). La sonie de sons purs partiellement masqués. Étude comparatif par une méthode d'égalisation et par la méthode des temps de réaction. Journal de Psychologie Normale et Pathologique, 63, 385-414.

Chocholle, R., \& Saulnier, C. (1969). Les temps de réaction à un son partiellement masqués par deux sons simultanés situés de part et d'autre du premier. Journal de Physiologie, Paris, 61(Suppl. 2), 246. (Abstract)

Emmerich, D. S., Pitchford, L. J., \& Becker, C. A. (1976). Reaction time to tones in tonal backgrounds and a comparison of reaction time to signal onset and offset. Perception \& Psychophysics, 20, $210-214$.

Fletcher, H., \& MUNSON, W. A. (1933). Loudness, its definition, measurement, and calculation. Journal of the Acoustical Society of America, 5, 82-108.

GREEN, D. M. (1967). Additivity of masking. Journal of the Acoustical Society of America, 41, 1517-1525.

SACHS, M. B., KIANG, N. Y.-S. (1968). Two-tone inhibition in auditory nerve fibers. Journal of the Acoustical Society of America, 43, 1120-1128.

WeGEL, R. L., \& LANE, C. E. (1924). The auditory masking of one pure tone by another and its probable relation to the dynamics of the inner ear. Physical Review, 23, 266-285.

\section{NOTE}

1. Since the timing of RT began with signal onset, some of the RTs may be those of second responses that occurred following a first response preceding the signal.

(Manuscript received July 20, 1987; revision accepted for publication May 18,1988 .) 